\title{
Influence of Dynamic Field of View Restrictions on Rotation Gain Perception in Virtual Environments
}

\author{
Hugo Brument ${ }^{1}$, Maud Marchal ${ }^{2}$, Anne-Hélène Olivier ${ }^{3}$, and Ferran Argelaguet ${ }^{1}$ \\ 1 Inria, Univ. Rennes, CNRS, IRISA, France \\ 2 Univ. Rennes, INSA, IRISA, Inria, CNRS - France and IUF \\ 3 Univ Rennes, Inria, CNRS, IRISA, M2S, Rennes, France \\ firstname.lastname@inria.fr
}

\begin{abstract}
The perception of rotation gain, defined as a modification of the virtual rotation with respect to the real rotation, has been widely studied to determine detection thresholds and widely applied to redirected navigation techniques. In contrast, Field of View (FoV) restrictions have been explored in virtual reality as a mitigation strategy for motion sickness, although they can alter user's perception and navigation performance in virtual environments. This paper explores whether the use of dynamic FoV manipulations, referred also as vignetting, could alter the perception of rotation gains during virtual rotations in virtual environments (VEs). We conducted a study to estimate and compare perceptual thresholds of rotation gains while varying the vignetting type (no vignetting, horizontal and global vignetting) and the vignetting effect (luminance or blur). 24 Participants performed 60 or 90 degrees virtual rotations in a virtual forest, with different rotation gains applied. Participants have to choose whether or not the virtual rotation was greater than the physical one. Results showed that the point of subjective equality was different across the vignetting types, but not across the vignetting effect or the turns. Subjective questionnaires indicated that vignetting seems less comfortable than the baseline condition to perform the task. We discuss the applications of such results to improve the design of vignetting for redirection techniques.
\end{abstract}

Keywords: Virtual Reality · Perception · Rotation Gains · Vignetting.

\section{Introduction}

Navigation is essential for exploring Virtual Environments (VEs). Then, it is important to provide to the users easy and comfortable navigation techniques for Virtual Reality (VR) experiences. While literature showed that real walking is the most ecological approach to navigate in VEs as it increases presence [59] and performance [32 48], the limitations of physical workspace in VR setups do not always enable users to walk. To encounter this constraint, numerous navigation techniques have been designed to freely navigate in VEs regardless of the size of the physical workspace [28]. Some encourage the physical movement of the user (e.g. redirected walking or walking-in-place), while others require minimal user motion, such as virtual steering techniques and teleport-based. However, virtual techniques lack of vestibular and proprioceptive feedback. 
Among the different navigation techniques, redirection techniques try to compensate the limited physical workspace while maintaining real walking to navigate in the VE [35]. Therefore they enable real walking while requiring minimal training to be used. They are based on (1) manipulating the users virtual and real paths and/or (2) manipulating the VE itself by changing its internal structure. One solution to achieved infinite walking in the VE in a limited work space was proposed by Razzaque [44] and named redirected walking. They added imperceptible yaw rotational gain (i.e. scaling the mapping between real and virtual motion) to user's view point in the Head Mounted Display (HMD) in order to subtlety reorient the user in the real environment.

Redirection techniques rely on detection thresholds (DTs) gains, which define the limit the user can detect or not the rotation gain. Numerous studies have been done to estimate the DTs of different types of gains such as rotation [11, 19, 54], translation [17, 33, 34] or curvature gains [5, 54]. In this paper, we will only focus on rotation gains. Imperceptibility of rotation gains for redirected walking implementations is a challenge and active topic of research in VR. When using rotation gains, VR designers have to be careful in their implementations of redirection techniques: they have to use gains that would be subtle enough in order to not not disturb users experience (high gains might be noticeable or make the navigation more difficult) and comfort (high gains may provoke more cybersickness). While research focused on how to increase the DTs without breaking presence, it is also important to consider the usability and factors that could influence the perception of rotation gains.

For instance, modern HMDs, such as the HTC Vive or the Oculus Rift, offer Field of Views (FoVs) up to 100 degrees. Recent work conducted with these HMDs has shown that FoV can alter motion perception [20,36]. However, related studies mostly focused on visually induced illusory self-motion known as vection [46, 47]. Little is known about the relation between the FoV and the perception of rotations gains in VEs. Some recent work showed differences between large and narrow FoV [61] on DTs, but no one explored the impact of dynamic FoV modifications on the perception of rotation gains. Such results could be important for VR developers to design new redirection techniques considering FoV restrictions for a wide audience since the FoVs vary between HMDs.

In this paper, we present a perceptual study assessing participants ability to discriminate changes between virtual and real rotations under different FoV restrictions, hereinafter referred as vignetting. Participants had to perform rotations in a virtual forest with different vignetting configurations (see Figure 1). Two factors were considered, the shape of the restriction (horizontal, global) and the visual effect (darkning and blur). We evaluated the participants perception of rotation gains by computing the Point of Subjective Equality (PSE) and the DTs for each condition. Our main hypothesis was that vignetting could reduce participants ability to determine whether a rotation gain was applied or not, therefore increasing the DTs. Our results contribute to the understanding of human perception in VEs and discuss the usability of vignetting for redirection techniques. 


\section{Related Work}

\subsection{Rotation Gains and Detection Thresholds}

In general, redirection techniques are required to scale users real movements in order to maintain them in the workspace. This intensity of the scaling is typically referred as gain. A rotation gain $g_{r} \in \mathbb{R}$ for head rotations is defined by the quotient between the virtual rotation $R_{\text {virtual }}$ and the physical (real-world) rotation $R_{\text {real }}: g_{r}=\frac{R_{\text {virtual }}}{R_{\text {real }}}$. Applying a rotation gain $g_{r}$ to $R_{\text {real }}$ will result to rotate the virtual camera by $R_{\text {virtual }} \times g_{r}$ instead of $R_{\text {real }}$. It means that if $g_{r}=1$, the virtual rotation remains the same than the real one. Otherwise, when $g_{r}>1$ or $g_{r}<1$, the virtual camera rotates respectively faster or slower than the user's head rotation. For example, applying a gain $g_{r}=2$ when the user rotates its head 90 degrees in the real world, the virtual camera rotates by 180 degrees [54]. Rotation gains can be applied for each angle of the rotation (i.e. pitch, yaw and roll). However, in redirection techniques, the gain is generally applied only on yaw rotations [19, 41, 44, 52]. Besides, the gain is mostly applied constantly during the whole rotation, but there exist also other implementations if the final rotation is known in advance [14,49, 63]. In this paper, we will only focus on amplified rotations with constant gains for yaw head rotations, excluding pitch or roll rotations [6].

Typically, perceptual studies to estimate detection thresholds (DTs) use two-alternative force-choice (2AFC) protocol, where different gains are applied, and estimate the detection thresholds by fitting a psychometric function to the percentage of positive answers. The gain at which the subject responds positively to the stimuli in $50 \%$ of the trials is defined as the Point of Subjective Equality (PSE), at which the user perceives the physical and the virtual rotations as identical. DTs are defined as the value of the gain at which the user has either $25 \%$ or $75 \%$ probability of choosing one item of the 2 AFC question. DTs interpretation can vary based on the gains used in the protocol. DTs then represent the boundaries at which the portion of incorrect $(25 \%$ DT) or correct $(75 \%$ DT) answers is significantly different from chance.

Amplified head rotations have been widely studied in VR [19,54] including different experimental conditions such as varying the amount of rotation to perform [11] (ranging from $10^{\circ}$ to $180^{\circ}$ ) ; adding visual effects [9, 39] (e.g. contrast inversion or sinus gratings) ; varying the gain implementation [14,63] (delaying the gain rotation based on the amount of rotation performed) ; using auditory cues [37,53] (specialized sound to redirected users) or distractors [41,61] (to lose focus on the gains); using different FoVs [6, 61] (e.g $40^{\circ}$ vs $110^{\circ}$ ) ; comparing perception of gain between a CAVE and a HMD [43] or different locomotion interfaces [10] (walking and wheelchair steering). These studies resulted in different PSE and thresholds values but in general, $25 \%$ and $75 \%$ thresholds ranged respectively between $0.59-0.93$ (25\% DT) and 1.10-1.27 (75\% DT), where the gains tested were between 0.5 and 1.5. Readers can refer to [25, 35] for further information about detection threshold of head rotation gains.

Literature showed that modifications of the FoV can alter motion perception. Yet, most of the presented studies did not restrict the participants' FoV. In the following section, we will introduce FoV restrictions in VR and studies its influence on participants' behavior. 


\subsection{Field of View and Vignetting in VR}

In human vision, the term "Field of View" (FoV) refers to the world that can be seen at any moment. It is defined as "the number of degrees of visual angle during stable fixation of the eyes" $|55|$. Humans effective visual field of view is $200^{\circ}$ horizontally and $150^{\circ}$ vertically [62]. Since vision is a fundamental cue for navigating, several research works conducted experiments to determine the effects of FoV restrictions on navigation performances. For instance, in Real Environments (REs), restricting both horizontal or vertical FoV increases the time to perform an obstacle course [18] 57]. Besides, the walking speed linearly decreases when the FoV is restricted [58].

In VR applications, the FoV refers rather to what is visible while wearing additional apparatus. Most of HMDs have limited FoVs ranging from $40^{\circ}$ to $110^{\circ}$ diagonal, which are considerably smaller than human FoV. There exist several ways in VR to restrict users virtual FoV by applying visual effects. The most common technique is called vignetting and consists in reducing the virtual camera's brightness or saturation toward the periphery compared to the virtual camera's center. Hence, it gradually reduces the users FoV by applying mostly a black color or some blur effects in the peripheral vision [12, 24]. Several vignetting models have been designed, either using a constant restriction [22], based on controller-based inputs [15,50], head movements [38] or ocular activity [1].

User studies have been conducted to determine whether vignetting could be a promising solution for decreasing cybersickness while preserving presence. However, the conclusions remain different across authors. Fernandes and Feiner assessed vignetting during navigation with a hand-held controller and they showed that vignetting can reduce cybersickness and improve users comfort [15]. Budhiraja et al. proposed a vignetting where mouse acceleration increased or decreased the Gaussian blur applied to the virtual camera [12]. Their vignetting allowed participants to experience less cybersickness during a first person shooter game in VR than the baseline group. Norouzi et al. studied the effect of vignetting during an exploration task in a virtual forest where head rotations gains were applied [38]. They found that most of the participants experienced more cybersickness with vignetting than without. These results could be explained by the difference in how users explored a VE using head movements (resulting in higher exploration) or a hand-held controller (resulting in slower exploration). Furthermore, vignetting seems not to have a negative effect on path integration [2], or spatial awareness [50]. Yet, some studies revealed that vignetting techniques are less preferred for navigation tasks [38, 50] where participants tend to prefer conditions without FoV restrictions and could lead to lower presence [29].

Rotation gains and vignetting have been widely studied but mostly separately. While the study of rotation gains was achieved to improve redirection techniques, and vignetting for improving user comfort and decrease cybersickness, little is known about the influence of vignetting on human perception. While Williams and Peck found that participant's ability to discriminate $90^{\circ}$ turn was more difficult (i.e. higher PSEs and DTs) with a wider FoV $\left(110^{\circ}\right)$ than a restricted one $\left(40^{\circ}\right)$ [61], we believe that restricting participants FoV could increase the DTs of rotation gains during turns. Indeed, Bolte et al. found that participants tend to underestimate pitch and roll gains when the FoV is reduced [6]. The objective of our study is therefore to use dynamic vignetting to explore 
its influence on rotation gain perception. We want to assess whether vignetting could increase DTs of rotation gains or not. Our main hypothesis is that FoV restrictions would alter participants ability to detect rotation gains. Our study aims at contributing to the improvement of redirection techniques.

\section{Dynamic Field of View Restrictions Design}

\subsection{Description}

In our experiment, we wanted to investigate the effect of several FoV restriction types. To design each vignetting, we followed models already designed to dynamically modify the FoV with respect to users head angular speed [4,38], but we adapted them in order to propose a generic vignetting model that allows any type of FoV restrictions and visual effects. Two different design choices were considered: (1) the restriction shape (i.e. the area of the FoV which is affected by the vignetting) ; (2) the effect type (i.e. the visual effect applied to the restricted area).

Regarding the restriction shape, most of studies used a circular restriction (an annulus defined by an inner and outer circles, hereafter referred as Global vignetting). In this paper, we also propose an Horizontal vignetting that reduces the users FoV to the opposite head rotation direction. The horizontal mode is inspired by the human anticipation behaviours in REs in which gaze will anticipate the head rotation during a turn [3]. Therefore, the Horizontal vignetting only hides the peripheral vision to the opposition gaze direction. The motivation to design this Horizontal vignetting is to reduce the amount of information and being more subtle than the Global one since the restriction is not applied in both eyes.

Regarding the effect type, we considered two methods that reduce the optical flow in the restricted area. A Luminance effect which decreases the contrast in the restricted area and a gaussian Blur effect which decreases visual saliency in the restricted area. While Luminance effect is the most widely used in VR applications using vignetting, we wanted to see whether Blur could provide similar perceptual results while less disturbing. This resulted in 4 different configurations: Global Luminance, Global Blur, Horizontal Luminance and Horizontal Blur (see Figure 1).

\subsection{Implementation Details}

Given a pixel position $p$ in normalized screen coordinates and the current restriction angle $\left(R_{t}\right)$, we first define whether the pixel falls within the restriction area:

$$
\operatorname{Shape}\left(p, R_{t}\right) \in[0,1]
$$

Zero means that the pixel is outside the restriction zone, one that the pixel is in the restriction zone and ]0,1 [ is transition zone. $R_{t}$ is defined by the yaw head rotation $w$ and Equation 1 has to be defined both for the Global, and the Horizontal restriction shapes.

The amount of restriction, $R_{t}$, is calculated using Equation 2 were $\alpha_{\max }$ and $\alpha_{\min }$ respectively represent the maximum and minimum values to apply the restriction. When 


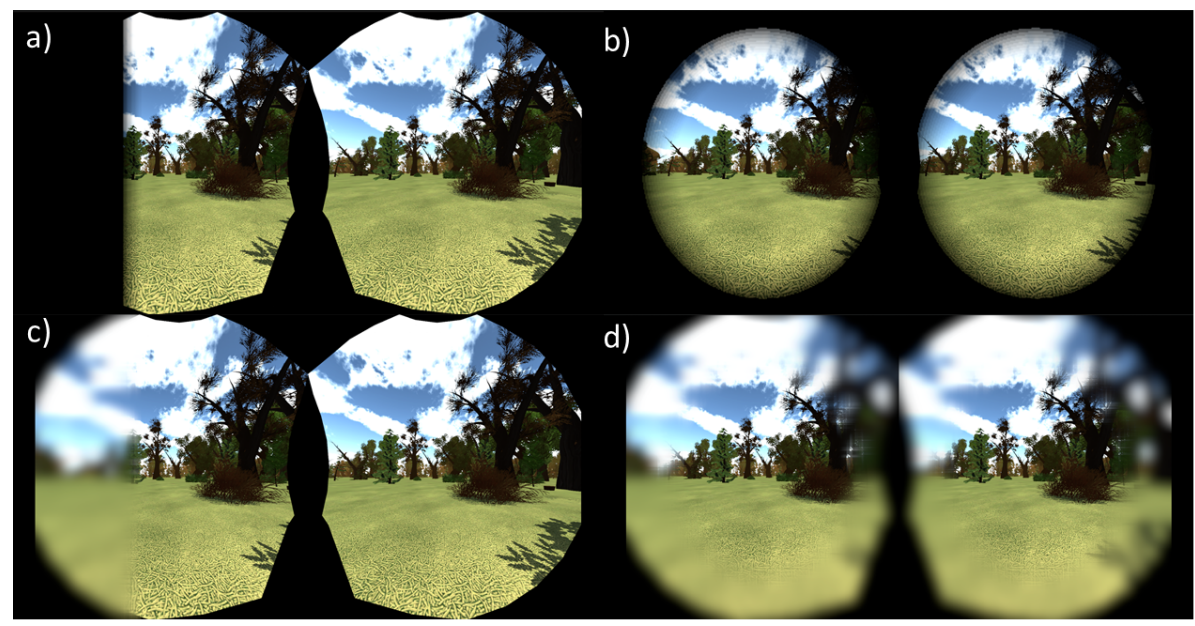

Fig. 1. Illustration of the 4 different FoV restrictions (vignetting) during the same rightwards rotation: (a) Horizontal Luminance ; (b) Global Luminance ; (c) Horizontal Blur ; (d) Global Blur.

$R_{t}(\omega)=\alpha_{\max }$ there is no restriction applied, and when $R_{t}(\omega)=\alpha_{\min }$ the restriction is maximal. Users FoV is reduced as $\omega$ increases.

$$
R_{t}(\omega)=\alpha_{\max }-\operatorname{Min}\left(\omega, \alpha_{\max }-\alpha_{\min }\right)
$$

In our model, $\alpha_{\max }$ and $\alpha_{\min }$ were respectively set to 56 and 18 degrees for the Horizontal restriction and 64 and 30 for the Global restriction. $\alpha_{\max }$ was defined considering the HMD used in the study (HTC Vive), while $\alpha_{\min }$ was defined empirically. For the Global restriction the minimum FoV was $60\left(\alpha_{\min } \times 2\right)$ while for the Horizontal restriction the minimum horizontal FoV was 74 degrees (asymmetric).

In order to decrease jitter for the head rotations speed, an hysteresis was applied based on the instantaneous head rotation speed $\left(\omega_{t}\right)$. We empirically found that $\gamma=0.4$ worked best to ensure that the FoV restriction would not jitter due to small head movements.

$$
\omega=\gamma * \omega_{t}
$$

Then, the cut-off of is defined by an inner and outer radius that together form an annulus for the Global type, and a rectangle for the Horizontal one. The opacity of the cut-off increases linearly from completely transparent to completely opaque (mask). The mask is calculated using Equation 4, while angle $(p)$ defines the viewing angle of the pixel $p$, and $\varepsilon_{\alpha}=10$ defines the transition zone.

$$
\operatorname{Shape}\left(p, R_{t}\right)=\frac{R_{t}-\operatorname{angle}(p)}{\varepsilon_{\alpha}} \in[0,1]
$$

Finally, we apply the restriction effect to the pixel, where $\operatorname{Shape}\left(p, R_{t}\right)$ defines the strength of the applied effect. The color of the pixel $p$ in normalized screen coordinates 
is computed as the linear interpolation between the pixel color and the visual effect (i.e. either the black color for the Luminance effect or the result of the gaussian blur for the Blur one) where the interpolant is the result of Shape $\left(p, R_{t}\right)$.

\section{User Study}

The goal of this experiment was to investigate the effect of vignetting on the detection threshold of amplified head rotations. We considered the previously defined vignetting types and effects. This experiment was inspired from similar protocol already performed to assess perception of rotation gains without [54] or with FoV restriction [61].

\subsection{Design and Hypotheses}

We conducted a 3 (Vignetting Type: none, horizontal, global) x 2 (Vignetting Effect: luminance, blur) x 2 (Rotation: $60^{\circ}, 90^{\circ}$ ) user study to estimate the perception threshold depending on the FoV restrictions. Vignetting Type and Rotation were within-participants factors whereas Vignetting Effect was a between-participant factor. We decided to test two different rotations because most of the studies only assessed $90^{\circ}$ turns, and Bruder et al. showed that perception of rotation gains can differ depending on the amount of rotation performed [11]. Besides, in navigation, shorter rotations than $90^{\circ}$ can occur and it is important to understand how participants could perceive gains during a shorter exposition.

For each Vignetting Type and Rotation, we tested 9 times each gain used in the experiment. The gains used in the experiment ranged from $1\left(90^{\circ}\right.$ physical rotation resulted in a $90^{\circ}$ virtual rotation) to $1.4\left(54^{\circ}\right.$ physical rotation resulted in a $90^{\circ}$ virtual rotation), incremented in steps of 0.1 . We only applied gain on the yaw axis. Excluding practice session, this resulted in 3 vignetting $\mathrm{x} 2$ rotations $\mathrm{x} 5$ gains $\mathrm{x} 9$ trials, totaling 270 trials per participant. The trials were randomized per block for each participant.

Note that, unlike similar protocols [11, 54,61], we did not assess gains below than 1 . These gains were not tested because our interest was to assess whether FOV manipulations could provide higher gains perception threshold. The task trials were a stimuli (gain applied) two-alternative forced choice (2AFC) task. 2AFC tasks avoid participant response bias as participants are forced to guess even when they are unsure of virtual head amplification. On average, when participants do not know the answer, if participants answer randomly, they will be correct $50 \%$ of the time. Our hypotheses for this experiment were:

- [H1] Detection thresholds would be higher when applying vignetting.

- [H2] Detection thresholds would differ depending on the vignetting effect.

- [H3] Detection thresholds would be higher for the $60^{\circ}$ turn than the $90^{\circ}$ one.

- [H4] Users would report no discomfort while using the FoV vignetting.

These hypotheses were motivated by our suggestions that vignetting could alter participants perception and therefore allow to add more imperceptible rotational gains. It means that we want to determine whether dynamic modification of FoV with different effects or restrictions could influence the way users perceive rotation with or without head amplifications. 


\subsection{Participants and Apparatus}

24 participants (18 males and 6 females) aged between 22 and 37 years old $(26.67 \pm 3.62$, mean \pm SD) without any ocular or locomotion disorders volunteered to this study. 14 participants reported using VR on a weekly or daily basis, 6 few times and 4 never. All participants except 4 had regular experiences with videos games. They were naive to the purpose of the experiment and signed an informed consent form. The study was conformed with the standards of the declaration of Helsinki.

We developed the application with Unity3D and we use a Vive Pro HMD, that has a resolution of 1440 x 1600 pixels per eye and a 110 degrees diagonal FoV. The reference coordinate system was defined by the HTC Vive tracking system. During the whole experiment we guaranteed the maximum frame-rate of the HTC Vive HMD $(90 \mathrm{~Hz})$. We use the Vive Wireless Adapter ${ }^{4}$ in order to prevent users from being bothered by cables, as it could potentially influence users behavior during their rotations.

The VE was a large outdoor forest with grass, trees and rocks. We designed it with the Green Forest Unity 3D asse 5 . This VE was chosen to generate motion flow from participants' while physically rotating. We also added a black cross located on the ground and a virtual sphere for calibration purposes.

\subsection{Procedure}

First, participants read and signed the consent form which provided detailed information regarding the experiment. They had a training session to get familiar with the task, the rotation gains, and the different vignetting conditions. Then, the experiment consisted in 9 randomized blocks, 3 for each vignetting (none, horizontal, global). Each block consisted in 30 trials ( 3 trials $x 5$ gains $x 2$ rotations), with a break after every 3 blocks completed. The experiment therefore resulted in a total of 270 trials ( 9 repetitions $x 3$ vignetting type $\mathrm{x} 5$ gains $\mathrm{x} 2$ rotations) per participants. At the beginning and the end of the experiment, participants filled a Simulator Sickness Questionnaire (SSQ) [21]. After filling the first demographic questionnaire (age, gender, amount of experience playing video games an exposure to VR), we assessed their dominant eye and measure their interpupillary distance (IPD). Then, they were placed at the center of the physical workspace and were equipped with the HMD and the controller.

A trial consisted of rotating the whole body in place (not just the head or the torso but also the feet) either 60 or $90^{\circ}$ clockwise or counter-clockwise. We randomly ordered clockwise and counter-clockwise rotations during the experiment. Participants could visualize the turn to perform thanks to an arrow indicating the rotation direction. Before starting the trial, they had to calibrate by looking at a red sphere that was displayed in front of them. Once they were staring at it, the sphere turned green and participants could press the controller's trigger to start the trial. Then, participants rotated until a red sphere appeared at the center of their vision, signaling that they should end their rotation by facing at this sphere until it turned green indicating successful trial completion. Participants had to confirm the trial by pressing the controller's trigger. If the participant

4 https://www.vive.com/eu/accessory/wireless-adapter/

5 https://assetstore.unity.com/packages/3d/environments/fantasy/green-forest-22762 
rotated past the virtual rotation, the green sphere's color changed to red and participants had to correct and maintain their orientation such that the sphere changed to blue green. At the end of the trial, the VE faded to black and participants had to answer the following 2AFC question: "My movement in the virtual world was greater than my physical movement: (yes or no answer)".

To prevent unintentional positional drift during the experiment, we ensure that the user started each trial around $50 \mathrm{~cm}$ to the center of the physical workspace, if the participants were not located nearby, they had to move towards a black cross displayed on the VE floor. Trials where the participants turned too quickly, slowly or inconsistently were rejected. For speed, participants were required to turn physically at between 45 and 180 degrees per second averaged across the entire turn. Trials were tested to ensure participants did not turn against the desired direction of motion. If a turn had failed, the trial would have been rejected and the $2 \mathrm{AFC}$ question would have been skipped and the participant got a feedback about the failure.

After a block of 30 trials, we asked participants to answer the following question "On a scale of $0-10,0$ being how you felt coming in, 10 is that you want to stop, where are you now?" [45]. This question ensured that participants did not feel severe sickness during the experiment, since doing a series of rotations with gains could lead to cybersickness. After every three blocks, the users took off the VR equipment and had a 5 minutes break to minimize potential negative effects of cybersickness.

At the end of the experiment, we asked participants to rate the comfort of each Vignetting Type to perform the task from 1 (not comfortable at all) to 7 (very comfortable). We also asked them to rank the Vignetting Type by their preferences (the one they preferred the most ranked 1st and the one they least preferred ranked 3rd). In total, the experiment took approximately an hour. At any time, users could ask for a break or stop the experiment.

\subsection{Data Analysis}

We recorded 6480 trials (24 users x 2 Vignetting Effects x 3 Vignetting Types x 5 Gains $x 9$ Repetitions) during this experiment. Practice trials before the experiment and between blocks were not included in the analysis. Preliminary data analysis revealed that there were no side effects between leftwards and rightwards rotations. We therefore mirrored the leftwards turns in order to remove the side factor from the analysis.

We computed for each participant the probability $P\left(g_{n} ; y e s\right)$ of responding "Yes" for a given gain to the question "My movement in the virtual world was greater than my physical movement", for each gain, turn and vignetting type. Then, a psychometric curve was fit to each participant's data and the Point of Subjective Equality (PSE), $25 \%$ and $75 \%$ threshold gains were computed with the Quickpsy package in R [30]. It fits by direct maximization of the likelihood psychometric functions of the form $\psi\left(g_{n}\right)=\gamma+(1-\gamma) * F(x)$, where $\gamma$ is the guess rate and $F$ the cumulative normal distribution function. We excluded 4 participants from the analysis because we were unable to fit a psychometric curve from their data (they mostly never answered "yes").

Before analyzing the positions and orientations of head and shoulders, we first resampled them and then applied a butterworth low-pass filter with a cutoff frequency of $1 \mathrm{~Hz}$ to remove oscillations due to the potential users displacements in the RE. We temporally 
normalized the evolution angular speed over the trials in order to analyze rotation behavior regarding the experimental conditions. We used the Statistical Parametric Mapping (SPM) method [16] to analyze the angular speed across the experimental conditions. This analysis allows comparing time-series data of different trials taking into account their variability at each time-step. In order to evaluate the effect of the Vignetting Effect, Vignetting Type, Rotation on PSEs and DTs, we performed a three-way analysis of variance (ANOVA) with repeated measures. We tested normal distribution of the data with the Shapiro-Wilk test. Greenhouse-Geisser adjustments to the degrees of freedom were applied, when appropriate, to avoid any violation of the sphericity assumption. Post-hoc analysis was based on pairwise t-tests with Bonferonni corrections. Only significant post-hoc comparison are reported in the next section. Finally, to analyse subjective data from the questionnaires, we used the Friedman test and post-hoc pairwise Wilcoxon tests with Bonferroni corrections.

\subsection{Results}

We found no significant effect of the vignetting type or gain on the evolution of the angular speed during a trial $(p>0.05)$. This means that participants rotation behavior remained similar across experimental conditions and trials (Figure 2). Besides, we noticed no effect of SSQ scores between the luminance and blur effects, and no fast SSQ average answers remained below 3 for each blocks during the experiment. These results are important for a fair comparison of DTs since the way participants perform the rotation and cybersickness could alter the perception of the rotation gains.

Psychometric curves were fit to the pooled results of participants data by Vignetting type, Vignetting Effect, and Rotation (Figure 33. Table 1 summarizes the PSEs and DTs computed for each experimental conditions based on participants individual fits. We compared $P\left(g_{n} ;\right.$ yes $)$, the probability of responding "yes" at a given gain $g_{n}$, with a 4-way ANOVA (Vignetting Type $\mathrm{x}$ Vignetting Effect $\mathrm{x}$ Rotation $\mathrm{x}$ Gain). There was a significant main effect of $\operatorname{Gain}\left(F(2.32,51.05)=177.68, p<.0001, \eta^{2}=.89\right)$, where post-hoc analyses showed that the higher the gain, the higher the probability of answer "greater" $(p<0.05)$.

To evaluate the effect of experimental conditions on PSEs, we performed a 3-way ANOVA (Vignetting Type $\mathrm{x}$ Vignetting Effect $\mathrm{x}$ Rotation). We found a significant effect of the Vignetting Type on the PSEs $\left(F(1.90,30)=3.99, p<0.05, \eta^{2}=.20\right)$ and DTs $\left(F(1.45,23.25)=8.11, p<0.01 \eta^{2}=.34\right)$, where post-hoc analyses showed that PSEs and DTs where higher with the Global vignetting than the baseline one (None). We found neither effect of Vignetting Effect $(p=0.41)$ nor Rotation $(p=0.13)$ on the PSEs and DTs.

A 2-way (Vignetting Type $x$ Vignetting Effect) ANOVA showed an effect of the Vignetting Type on comfort $\left(F(1.30,20.74)=5.00, p<0.05, \eta^{2}=.24\right)$, where Global vignetting was less comfortable than the None and the Horizontal ones $(p<0.05)$. Figure 4 shows the number of votes regarding vignetting type preferences (the most preferred ranked 1 st and the least preferred ranked 3rd). A chi-square test showed that the Vignetting Effects were not independent $\left(\chi^{2}(8)=18.595, p<0.05\right)$. In overall, participants ranked the baseline (None, no vignetting) as the most preferred then the Horizontal and finally the Global. 
60

Color

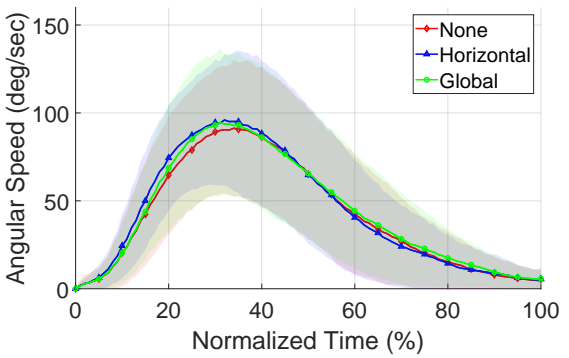

Blur

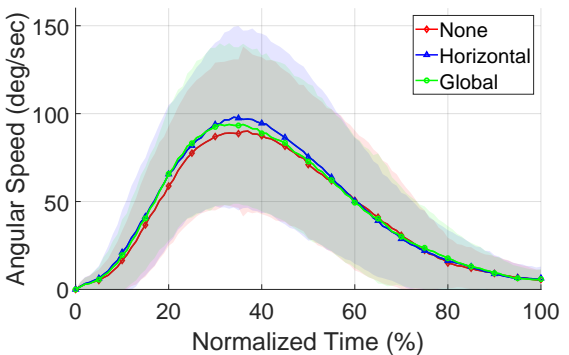

90
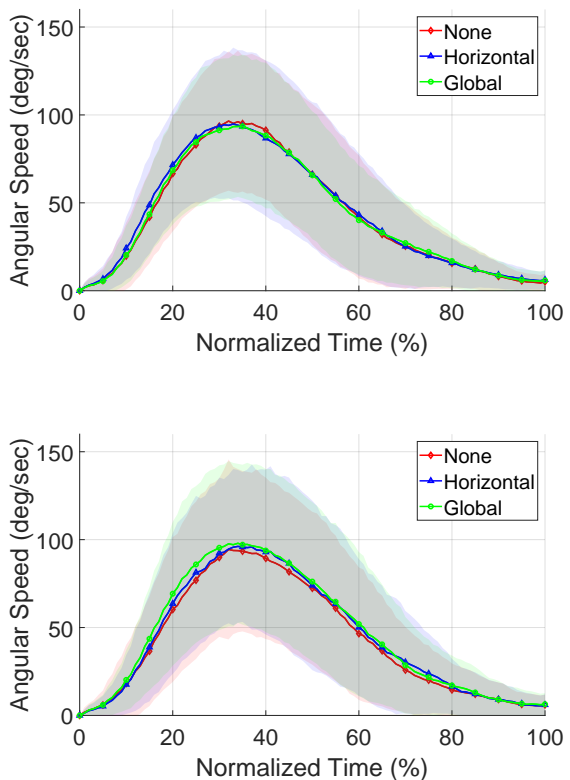

Fig. 2. This figure shows typical temporal evolution of mean and standard deviation of angular speed for each Vignetting type (None in red, Horizontal in blue and Global in green), Vignetting Effect (Color on first row and Blur on second) during $60^{\circ}$ and $90^{\circ}$ turns. Each sample of the temporal sequence is a dependant variable. No effect was found across the conditions.

Table 1. The 25\%, PSE, and 75\% threshold gains derived from the psychometric curves. Results are grouped by Vignetting Effect, Type and Rotation.

\begin{tabular}{|c|c|c|c|c|c|c|c|}
\hline \multirow[t]{3}{*}{ Effect } & \multirow[t]{3}{*}{ Type } & \multicolumn{6}{|c|}{ Rotation } \\
\hline & & \multicolumn{3}{|c|}{60} & \multicolumn{3}{|c|}{90} \\
\hline & & $25 \%$ & PSE & $75 \%$ & $25 \%$ & PSE & $75 \%$ \\
\hline Color & None & $1.13(0.11)$ & $1.23(0.10)$ & $1.32(0.11)$ & $1.15(0.05)$ & $1.24(0.05)$ & $1.33(0.06)$ \\
\hline Blur & None & $1.11(0.08)$ & $1.20(0.07)$ & $1.29(0.06)$ & $1.15(0.11)$ & $1.22(0.08)$ & $1.30(0.07)$ \\
\hline \multirow{2}{*}{ Color } & Horizontal & $1.13(0.13)$ & $1.23(0.08)$ & $1.32(0.09)$ & $1.12(0.08)$ & $1.25(0.04)$ & $1.40(0.09)$ \\
\hline & Global & $1.13(0.06)$ & $1.25(0.08)$ & $1.38(0.12)$ & $1.16(0.07)$ & $1.26(0.06)$ & $1.35(0.08)$ \\
\hline \multirow{2}{*}{ Blur } & Horizontal & $1.10(0.10)$ & $1.20(0.09)$ & $1.29(0.07)$ & $1.13(0.08)$ & $1.24(0.05)$ & $1.35(0.07)$ \\
\hline & Global & $1.08(0.11)$ & $1.20(0.07)$ & $1.35(0.06)$ & $1.12(0.12)$ & $1.24(0.07)$ & $1.33(0.07)$ \\
\hline
\end{tabular}




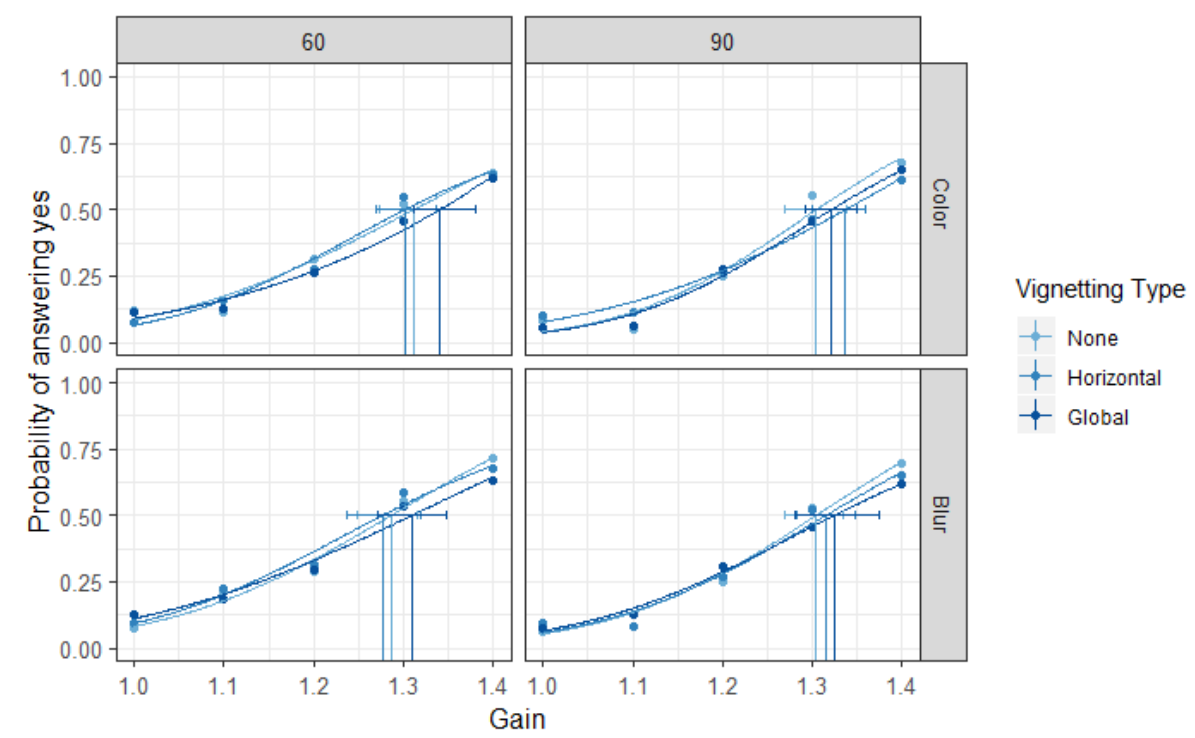

Fig. 3. Psychometric functions computed from the pooled results for each Vignetting Type (None in red, Horizontal in green, Global in blue). The $\mathrm{x}$-axis shows the gain applied and the $y$-axis the probability of answering "yes" to the question "My movement in the virtual world was greater than my physical movement". Results are grouped by Rotation $(60,90)$ and Vignetting Effect (Color, Blur).

\section{Discussion}

Our main objective was to assess whether vignetting (either its type or its effect) could alter the perception of rotation gains in virtual environments. More precisely, we designed an experiment where participants had to perform a 60 or 90 degrees turns where we applied different rotation gains (from 1 to 1.4) and vignetting (None, Horizontal, Global). We analyzed participants ability to detect or not the gains by computing their PSEs and DTs. While we observed an effect of the Vignetting Type on the PSEs variable of the experiment, our results showed that the average values remain similar.

Regarding [H1], we were expecting that restricting the participants FoV by applying a vignetting would make the detection of rotation gains more difficult, resulting in higher PSEs and DTs. This hypothesis was motivated by the fact that peripheral vision could help to disambiguate the perception of self motion [27]. We extended this statement by supposing peripheral vision could also help detecting or not a rotation gain. We wanted therefore to check how users would be able to determine a rotation gain with less information of the VE. Even though we found an effect of the Vignetting Type on the PSEs and DTs, Table 1 shows that the average PSEs per condition remained quite similar (around 1.20 and 1.26). We guaranteed in our vignetting model that the amount of restriction between the Horizontal and Global was similar, but the restricted regions were different. Restricting both eyes (Global vignetting) seemed be more efficient 


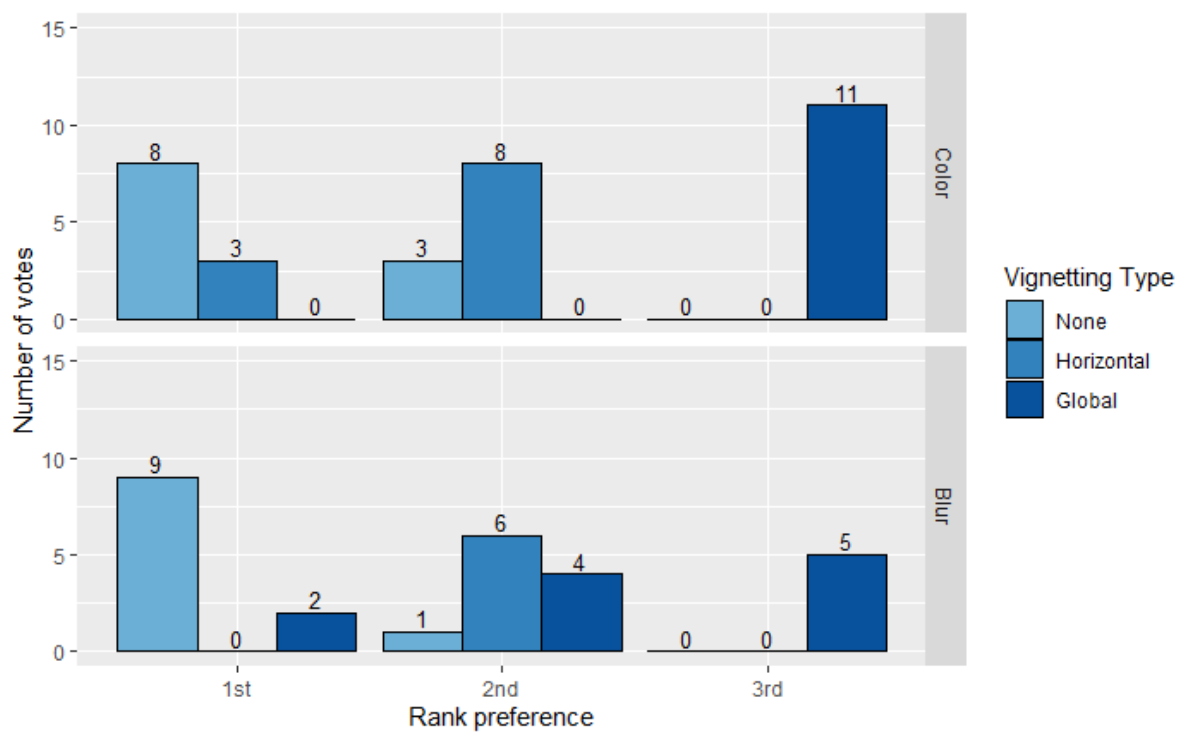

Fig. 4. Distribution of participants vignetting preferences (grouped by Vignetting Effect). At the end of the experiment, we asked participants to rank from their most to their least preferred vignetting.

for disturbing the detection of rotation gains than restricting the eye opposite to the participant's rotation (Horizontal vignetting). One explanation could be that, during the rotation, the eyes were staring at the opposite direction of the region where the Horizontal vignetting was applied, while the Global one also covered peripheral region opposite to the rotation's direction. Then, Global vignetting tended to be more efficient in disturbing the detection of rotation gains than the the others conditions.

In our experiment, we had the Vignetting Effect as a between group variable. Half of participants tested the Luminance effect and the other half the Blur one. We wanted to see whether different visual effects could alter perception of gains, as it was demonstrated that they can alter users self motion perception [9]. We did not see differences between both effects, rejecting [H2]. While most of the vignetting effects used in VR applications are based on a black texture decreasing the contrast in the restricted area, we wanted to see whether a blurring effect, that could be less noticeable to the user, could provide similar or higher PSEs and DTs. Finally, our experiment showed that both visual effects were similar. We could therefore consider different effects with respect to users preferences.

While most studies on the perception of rotation gains considered mainly $90^{\circ}$ rotations, we wanted to see if the detection of rotation gains could be more difficult with a shorter rotation $\left(60^{\circ}\right.$ in our experiment). With [H3], we expected differences in PSEs and DTs between both turns. Bruder et al. showed that participants were better at discriminating rotations when the virtual turning angle is rather large [11]. Even though we did not find a significant effect of Rotation on PSEs and DTs, Figure 3 shows that, for $60^{\circ}$ turns, the PSEs for the Global Vignetting Type is higher than the two others for both 
Vignetting Effects (the blue curve is slightly shifted to the right compared to the others). Thus, the use of vignetting and rotation gains might be interesting to manipulate user rotations during shorter rotations than $90^{\circ}$.

Subjective questionnaires showed that users preferred to perform the rotation task without vignetting. While some research work showed the benefits of vignetting to reduce cybersickness [15], participants did not prefer the use of vignetting to perform navigation tasks [38, 50]. Thus, it is hard to determine the benefits of vignetting regarding user preferences and we believe that vignetting based on head movements is not appropriate for all users in VR. For instance, all participants that reported using VR on a weekly or daily basis noticed both vignetting effects, while the participants that experienced VR for the first time did not notice them. Besides, most of the "expert" participants did not recommend the vignetting effects, reporting that it was too constraining and uncomfortable. Few of them also reported that they were not affected by the vignetting since they were focused on the detection task. Thus, it could be interesting to consider the vignetting with respect to the user VR experience. Our results demonstrated the opposite of our hypothesis [H4]. We could have expected that the blurring effect (that mimicries the natural blurring in the peripheral vision) would be more comfortable than the luminance one (that hides the peripheral vision). However, the Horizontal vignetting seemed more appreciated by participants than the Global vignetting. One reason could be that it was less noticeable, thus participants were less bothered during the tasks.

The literature notes that during a sensory conflict between visual and vestibular cues, the visual information is predominant on the vestibular and the proprioceptive ones during locomotion. During the task, participants had to compare their perceived virtual rotation with their real rotation. The vignetting restricted information in the peripheral region, generating less visual information than in the baseline condition. Yet, since the rotations asked were constant, the amount of extra-retinal information received by participants remained the same across vignetting conditions (i.e. constant optical flow). Then, we believe that the similar PSEs and DTs across conditions can be explained by three external factors that we could explore in future experiments:

1. Optic flow can be used to control heading direction [60] while walking. Research work showed that offsetting the location of the Focus of Expansion (FoE) alters gait behavior, resulting in a walking path that is deviated at in a direction opposite to the FoE [51,60]. Besides, asymmetric optic flow can alter the steering behavior, some studies showed that when there is an inconsistency between the speed of two corridors' walls, the chosen trajectory is the one that reduces the difference between those regions (participants drift towards the slower moving wall) [13. 23]. Thus, manipulation of optic flow could alter the perception of rotation gains. It can be interesting to have a look at these manipulations since optic flow is a major component in the perception of self motion. Besides, in our experiment, as only rotation was considered, the optic flow was constant for each pixel. Introducing a translation component could help to determine whether the amount of optical flow could disturb the detection of rotational gains or not.

2. Saccades (and vestibulo-ocular-reflex) could contribute to the detection of rotation gains. Saccadic suppression of image has been already used to subtlety reorient participants in the VE. They take advantage of the inability to detect changes in 
the location of a target when the change occurs immediately before, during or shortly after the saccade [8]. Bolte and Lappe suggested that participants are more sensitive to scene rotations orthogonal to the saccade than in the same direction of this saccade [7]. Sun et al. implemented a redirected walking controller that rotates up to 0.14 degrees/frame the virtual camera when a saccade is detected [56]. Moreover, Langbehn et al. assessed the threshold of translation and rotations offset during participants blinks [26]. They reported that it is possible to apply a $+/-5^{\circ}$ reorientation in the transverse plane along the line of gaze during saccades $>15^{\circ}$ (and users tend to fail detecting translations shift from range 4-9 $\mathrm{cm}$ ). Therefore, it is easier to apply a gain during a saccade than during a fixation. In our experiment, participants had to stare at a sphere at the end of the rotation task. When a gain was applied, the mismatch between the gaze direction and the sphere position in the VE might have been noticeable and therefore have contributed to detect the gain. Recording gaze activity during such experiment would help to determine whether gaze behavior is different according to the gain.

3. Proprioception is an important cue while navigating. In our experiment, participants could have relied on computing the amount of rotation done in RE (with their feet orientation) and check if it matches with the final orientation in the VE. Marlinsky showed that blindfolded people tend to overestimate rotations of lower magnitudes and underestimate those of higher magnitudes [31]. Besides, they overestimated passive rotations and this estimation was linearly related to to the magnitude of turn. Research work showed the importance of neck proprioception in the perception of body orientation and motion [40, 42]. It may be difficult to assess the impact of proprioception because we cannot isolate this factor. We could, for instance, ask participants the amount of physical rotation they performed at each trial and measure the rotation error with and without gain. We could then see whether the proprioception information was prior to the visual one or not.

\section{Limitations and Future Work}

In our experiment, we only used gains above 1 because we wanted to assess whether we can increase rotation gains with vignetting. However, this choice could have led to an asymmetry that could have biased our results (and therefore having an overestimation of the PSEs and DTs) since a gain was applied in $80 \%$ of the trials (i.e. answering "yes" to the 2AFC question). Besides, participants had to discriminate real and virtual rotations during a single turn. However, some studies showed that PSEs and DTs can differ according to the rotation task (e.g. discrimination between (1) virtual and physical rotation and (2) two successive rotations) [54]. Thus, further experiments are required to assess gains below 1 and varying the rotation task.

The absence of significant differences between the FoV Vignetting Types could be linked to the vignetting model itself. Indeed, its design was based on previous models of the literature, and we respected the maximum of contraction used in most of VR applications. Yet, further work is required to determine how we could improve the vignetting so that it will be adapted to the user and could potentially increase the DTs for redirection techniques in VR. 
Finally, other factors might impact the perception of rotation gains in VEs (e.g tangential and angular speeds). For example, Neth et al. investigated the influence of walking speed on the detection of curvature gain [33] and demonstrated that people are significantly less sensitive towards walking on a curved path when walking slower. Further experiment would be needed to assess the perception of rotation gains during virtual translations with different angular speeds.

\section{Conclusion}

In this paper, we proposed to study the impact of different vignetting implementations on the perception of rotation gains during virtual turns in VE. The results of our experiment showed a difference of the Vignetting Type on the PSEs and DTs, but no effect for the Vignetting Effect nor Rotation. Yet, the average PSEs and DTs remained quite similar across the different conditions, and the results of our experiment might suggest that vignetting could not necessarily alter the perception of rotation gains. It is difficult to conclude that our results encourage the use of vignetting in order to increase the gains used in redirected techniques, since they allow only slight increase of rotation gains while altering users comfort. Nevertheless, we believe that the use of FoV manipulations could be considered as an interesting option for VR applications. Vignetting should therefore be designed with a user-centered approach in order to make it affordable in redirection techniques implementations.

\section{References}

1. Adhanom, I.B., Griffin, N.N., MacNeilage, P., Folmer, E.: The effect of a foveated field-ofview restrictor on vr sickness. In: Proc. of IEEE Conference on Virtual Reality and 3D User Interfaces (VR). pp. 645-652 (2020)

2. Al Zayer, M., Adhanom, I.B., MacNeilage, P., Folmer, E.: The effect of field-of-view restriction on sex bias in vr sickness and spatial navigation performance. In: Proc. of the ACM CHI Conference on Human Factors in Computing Systems. p. 354 (2019)

3. Bernardin, D., Kadone, H., Bennequin, D., Sugar, T., Zaoui, M., Berthoz, A.: Gaze anticipation during human locomotion. Experimental Brain Research 223(1), 65-78 (2012)

4. Bolas, M., Jones, J.A., McDowall, I., Suma, E.: Dynamic field of view throttling as a means of improving user experience in head mounted virtual environments (May 9 2017), uS Patent $9,645,395$

5. Bölling, L., Stein, N., Steinicke, F., Lappe, M.: Shrinking circles: Adaptation to increased curvature gain in redirected walking. IEEE Transactions on visualization and computer graphics 25(5), 2032-2039 (2019)

6. Bolte, B., Bruder, G., Steinicke, F., Hinrichs, K., Lappe, M.: Augmentation techniques for efficient exploration in head-mounted display environments. In: Proceedings of the 17th ACM Symposium on Virtual Reality Software and Technology. pp. 11-18 (2010)

7. Bolte, B., Lappe, M.: Subliminal reorientation and repositioning in immersive virtual environments using saccadic suppression. IEEE Transactions on visualization and computer graphics 21(4), 545-552 (2015)

8. Bridgeman, B., Hendry, D., Stark, L.: Failure to detect displacement of the visual world during saccadic eye movements. Vision research 15(6), 719-722 (1975) 
9. Bruder, G., Steinicke, F., Wieland, P., Lappe, M.: Tuning Self-Motion Perception in Virtual Reality with Visual Illusions. IEEE Transactions on Visualization and Computer Graphics 18(7), 1068-1078 (Jul 2012). https://doi.org/10.1109/TVCG.2011.274

10. Bruder, G., Interrante, V., Phillips, L., Steinicke, F.: Redirecting walking and driving for natural navigation in immersive virtual environments. IEEE Transactions on visualization and computer graphics 18(4), 538-545 (2012)

11. Bruder, G., Steinicke, F., Hinrichs, K.H., Lappe, M.: Reorientation during body turns. In: Proc. of EGVE/ICAT/EuroVR. pp. 145-152 (2009)

12. Budhiraja, P., Miller, M.R., Modi, A.K., Forsyth, D.: Rotation blurring: use of artificial blurring to reduce cybersickness in virtual reality first person shooters. arXiv preprint arXiv:1710.02599 (2017)

13. Chou, Y.h.: Effects of symmetric and asymmetric optic flow speed manipulations on locomotion in younger and older adults. Ph.D. thesis, Boston University (2005)

14. Congdon, B.J., Steed, A.: Sensitivity to rate of change in gains applied by redirected walking. In: Proc. of the 25th ACM Symposium on Virtual Reality Software and Technology. p. 3 (2019)

15. Fernandes, A.S., Feiner, S.K.: Combating vr sickness through subtle dynamic field-of-view modification. In: Proc. of IEEE Symposium on 3D User Interfaces (3DUI). pp. 201-210 (2016)

16. Friston, K., Ashburner, J., Kiebel, S., Nichols, T., Penny, W.: Statistical Parametric Mapping. Academic Press (2007)

17. Hopper, J.E., Finney, H., Jones, J.A.: Field of view and forward motion discrimination in virtual reality. In: Proc. of IEEE Conference on Virtual Reality and 3D User Interfaces (VR). pp. 1663-1666 (2019)

18. Jansen, S.E.M., Toet, A., Delleman, N.J.: Restricting the vertical and horizontal extent of the field-of-view: effects on manoeuvring performance. The Ergonomics Open Journal 3(1) (2010)

19. Jerald, J., Peck, T., Steinicke, F., Whitton, M.: Sensitivity to scene motion for phases of head yaws. In: Proceedings of the 5th ACM symposium on Applied perception in graphics and visualization. pp. 155-162 (2008)

20. Jones, J.A., Swan II, J.E., Bolas, M.: Peripheral stimulation and its effect on perceived spatial scale in virtual environments. IEEE Transactions on visualization and computer graphics 19(4), 701-710 (2013)

21. Kennedy, R.S., Lane, N.E., Berbaum, K.S., Lilienthal, M.G.: Simulator sickness questionnaire: An enhanced method for quantifying simulator sickness. The International Journal of Aviation Psychology 3(3), 203-220 (1993)

22. Kopper, R., Stinson, C., Bowman, D.: Towards an understanding of the effects of amplified head rotations. In: Proc. of the 3rd IEEE VR Workshop on Perceptual Illusions in Virtual Environments. vol. 2 (2011)

23. Kountouriotis, G.K., Shire, K.A., Mole, C.D., Gardner, P.H., Merat, N., Wilkie, R.M.: Optic flow asymmetries bias high-speed steering along roads. Journal of Vision 13(10), 23-23 (2013)

24. Langbehn, E., Raupp, T., Bruder, G., Steinicke, F., Bolte, B., Lappe, M.: Visual blur in immersive virtual environments: does depth of field or motion blur affect distance and speed estimation? In: Proceedings of the 22nd ACM Conference on Virtual Reality Software and Technology. pp. 241-250 (2016)

25. Langbehn, E., Steinicke, F.: Redirected Walking in Virtual Reality, pp. 1-11. Springer International Publishing, Cham (2018)

26. Langbehn, E., Steinicke, F., Lappe, M., Welch, G.F., Bruder, G.: In the blink of an eye: Leveraging blink-induced suppression for imperceptible position and orientation redirection in 
virtual reality. ACM Trans. Graph. 37(4) (Jul 2018). https://doi.org/10.1145/3197517.3201335 https://doi.org/10.1145/3197517.3201335

27. Lappe, M., Bremmer, F., Van den Berg, A.: Perception of self-motion from visual flow. Trends in cognitive sciences 3(9), 329-336 (1999)

28. LaViola, J.J., Kruijff, E., McMahan, Ryan P. Bowman, D.A., Poupyrev, I.: 3D User Interfaces: Theory and Practice. Addison Wesley Longman Publishing Co., Inc. (2017)

29. Lin, J.J.., Duh, H.B.L., Parker, D.E., Abi-Rached, H., Furness, T.A.: Effects of field of view on presence, enjoyment, memory, and simulator sickness in a virtual environment. In: Proc. of IEEE Virtual Reality Conference. pp. 164-171 (2002)

30. Linares, D., Lopez-Moliner, J.: quickpsy: An $r$ package to fit psychometric functions for multiple groups. The R Journal 8, 122-131 (2016)

31. Marlinsky, V.: Vestibular and vestibulo-proprioceptive perception of motion in the horizontal plane in blindfolded man-ii. estimations of rotations about the earth-vertical axis. Neuroscience 90(2), 395-401 (1999)

32. Nabiyouni, M., Saktheeswaran, A., Bowman, D.A., Karanth, A.: Comparing the performance of natural, semi-natural, and non-natural locomotion techniques in virtual reality. In: Proc. of IEEE Symposium on 3D User Interfaces (3DUI). pp. 3-10 (2015)

33. Neth, C.T., Souman, J.L., Engel, D., Kloos, U., Bulthoff, H.H., Mohler, B.J.: Velocitydependent dynamic curvature gain for redirected walking. IEEE Transactions on visualization and computer graphics 18(7), 1041-1052 (2012)

34. Nguyen, A., Cervellati, F., Kunz, A.: Gain compensation in redirected walking. In: Proc. of the 23rd ACM Symposium on Virtual Reality Software and Technology. p. 20 (2017)

35. Nilsson, N.C., Peck, T., Bruder, G., Hodgson, E., Serafin, S., Whitton, M., Steinicke, F., Rosenberg, E.S.: 15 years of research on redirected walking in immersive virtual environments. IEEE Computer Graphics and Applications 38(2), 44-56 (Mar 2018)

36. Nilsson, N.C., Serafin, S., Nordahl, R.: Establishing the range of perceptually natural visual walking speeds for virtual walking-in-place locomotion. IEEE Transactions on visualization and computer graphics 20(4), 569-578 (2014)

37. Nilsson, N.C., Suma, E., Nordahl, R., Bolas, M., Serafin, S.: Estimation of detection thresholds for audiovisual rotation gains. In: Proc. of IEEE Virtual Reality (VR). pp. 241-242 (2016)

38. Norouzi, N., Bruder, G., Welch, G.: Assessing vignetting as a means to reduce vr sickness during amplified head rotations. In: Proceedings of the 15th ACM Symposium on Applied Perception. p. 19 (2018)

39. Paludan, A., Elbaek, J., Mortensen, M., Zobbe, M., Nilsson, N.C., Nordahl, R., Reng, L., Serafin, S.: Disguising rotational gain for redirected walking in virtual reality: Effect of visual density. In: Proc. of IEEE Virtual Reality (VR). pp. 259-260 (2016)

40. Panichi, R., Botti, F.M., Ferraresi, A., Faralli, M., Kyriakareli, A., Schieppati, M., Pettorossi, V.E.: Self-motion perception and vestibulo-ocular reflex during whole body yaw rotation in standing subjects: the role of head position and neck proprioception. Human movement science 30(2), 314-332 (2011)

41. Peck, T.C., Fuchs, H., Whitton, M.C.: Evaluation of reorientation techniques and distractors for walking in large virtual environments. IEEE Transactions on Visualization and Computer Graphics 15(3), 383-394 (2009)

42. Pettorossi, V.E., Schieppati, M.: Neck proprioception shapes body orientation and perception of motion. Frontiers in Human Neuroscience 8, 895 (2014)

43. Ragan, E.D., Scerbo, S., Bacim, F., Bowman, D.A.: Amplified head rotation in virtual reality and the effects on $3 \mathrm{~d}$ search, training transfer, and spatial orientation. IEEE Transactions on visualization and computer graphics 23(8), 1880-1895 (2016)

44. Razzaque, S., Kohn, Z., Whitton, M.C.: Redirected walking. Tech. rep., Department of Computer Science, University of North Carolina, Chapel Hill, North Carolina, USA (2001) 
45. Rebenitsch, L., Owen, C.: Individual variation in susceptibility to cybersickness. In: Proc. of the 27th annual ACM symposium on User interface software and technology. pp. 309-317 (2014)

46. Riecke, B.E., Schulte-Pelkum, J., Avraamides, M.N., von der Heyde, M., Bülthoff, H.H.: Scene consistency and spatial presence increase the sensation of self-motion in virtual reality. In: Proc. of the 2nd Symposium on Applied Perception in Graphics and Visualization. pp. $111-118$ (2005)

47. Riecke, B.E., Schulte-Pelkum, J., Avraamides, M.N., Heyde, M.V.D., Bülthoff, H.H.: Cognitive factors can influence self-motion perception (vection) in virtual reality. ACM Transactions on Applied Perception (TAP) 3(3), 194-216 (2006)

48. Ruddle, R.A., Volkova, E., Bülthoff, H.H.: Learning to Walk in Virtual Reality. ACM Transactions on Applied Perception 10(2), 11:1-11:17 (2013)

49. Sargunam, S.P., Moghadam, K.R., Suhail, M., Ragan, E.D.: Guided head rotation and amplified head rotation: Evaluating semi-natural travel and viewing techniques in virtual reality. In: 2017 IEEE Virtual Reality (VR). pp. 19-28. IEEE (2017)

50. Sargunam, S.P., Ragan, E.D.: Evaluating joystick control for view rotation in virtual reality with continuous turning, discrete turning, and field-of-view reduction. In: Proceedings of the 3rd International Workshop on Interactive and Spatial Computing. pp. 74-79. ACM (2018)

51. Sarre, G., Berard, J., Fung, J., Lamontagne, A.: Steering behaviour can be modulated by different optic flows during walking. Neuroscience letters 436(2), 96-101 (2008)

52. Schmitz, P., Hildebrandt, J., Valdez, A.C., Kobbelt, L., Ziefle, M.: You Spin my Head Right Round: Threshold of Limited Immersion for Rotation Gains in Redirected Walking. IEEE Transactions on Visualization and Computer Graphics 24(4), 1623-1632 (2018)

53. Serafin, S., Nilsson, N.C., Sikstrom, E., De Goetzen, A., Nordahl, R.: Estimation of detection thresholds for acoustic based redirected walking techniques. In: Proc. of IEEE Virtual Reality (VR). pp. 161-162 (2013)

54. Steinicke, F., Bruder, G., Jerald, J., Frenz, H., Lappe, M.: Estimation of detection thresholds for redirected walking techniques. IEEE Transactions on Visualization and Computer Graphics 16(1), 17-27 (2010)

55. Strasburger, H., Pöppel, E.: Visual field. Encyclopedia of neuroscience pp. 2127-2129 (2002)

56. Sun, Q., Patney, A., Wei, L.Y., Shapira, O., Lu, J., Asente, P., Zhu, S., McGuire, M., Luebke, D., Kaufman, A.: Towards virtual reality infinite walking: dynamic saccadic redirection. ACM Transactions on Graphics (TOG) 37(4), 1-13 (2018)

57. Toet, A., van der Hoeven, M., Kahrimanović, M., Delleman, N.J.: Effects of field of view on human locomotion. In: Head-and Helmet-Mounted Displays XIII: Design and Applications. vol. 6955, p. 69550H. International Society for Optics and Photonics (2008)

58. Toet, A., Jansen, S.E., Delleman, N.J.: Effects of field-of-view restrictions on speed and accuracy of manoeuvring. Perceptual and motor skills 105(3_suppl), 1245-1256 (2007)

59. Usoh, M., Arthur, K., Whitton, M.C., Bastos, R., Steed, A., Slater, M., Brooks, Jr., F.P.: Walking $>$ walking-in-place $>$ flying, in virtual environments. In: Proc. of the 26th Annual Conference on Computer Graphics and Interactive Techniques. pp. 359-364 (1999)

60. Warren Jr, W.H., Kay, B.A., Zosh, W.D., Duchon, A.P., Sahuc, S.: Optic flow is used to control human walking. Nature neuroscience 4(2), 213 (2001)

61. Williams, N.L., Peck, T.C.: Estimation of rotation gain thresholds considering fov, gender, and distractors. IEEE Transactions on visualization and computer graphics 25(11), 3158-3168 (2019)

62. Wolpert, L., Warren, R., Wertheim, A.: Field-of-view information for self-motion perception. Erlbaum Hillsdale, NJ (1990)

63. Zhang, R., Kuhl, S.A.: Human sensitivity to dynamic rotation gains in head-mounted displays. In: Proceedings of the ACM Symposium on Applied Perception. p. 71-74 (2013) 